\author{
Robert J. Melosh \\ Department of Civil and \\ Environmental Engineering \\ Duke University \\ Durham, NC 27706
}

\title{
Modeling Accuracy in FEA of Vibrations of a Drumhead
}

\begin{abstract}
The study of the problem of predicting values of Rayleigh's quotient for a square drumhead provides a basis for assessing the relation between grid size, accuracy of analysis results, and efficiency of data processing in finite element analysis. The analysis data indicate that unacceptable grid sampling can occur even for the fine grids, that strictly monotonic convergence is attainable for vibration analysis, and that more efficient computer analysis associates with use of curve fitting analysis of conventional finite element analysis results. (c) 1993 John Wiley \& Sons, Inc.
\end{abstract}

\section{INTRODUCTION}

Finite element analysis modeling approximations are the inaccuracies introduced in the numerical model that vanish as the grid interval approaches zero. Modeling approximations include the definition of the original and deformed geometry of the structure, the equations and coefficients of the material constitutive model, and the statement of the problem boundary conditions.

Consider the structural configuration defined by Figure 1. This thin, square-shaped flat surface is pinned along its outer boundary. The uniformly thick surface has a uniform mass density. The membrane is stretched over a square frame, resulting in a uniform tensile force in the membrane. We focus on the relation between the grid interval, accuracy, and the number of calculations required in predicting the Rayleigh quotients (RQ) of the drumhead of Figure 1.

Limiting our attention to the linearized equations of equilibrium and isotropic homogeneous material behavior leads to the differential equation given by Young (1962):

$$
d^{2} w /\left(d x^{2}\right)+d^{2} w /\left(d y^{2}\right)=d^{2} w /\left(d t^{2}\right) \mu / N
$$

where $w$ is the lateral displacement and $x$ and $y$ are the coordinates along the $x$ and $y$ axes, $t$ is the time variable, $\mu$ is the mass density, and $N$ is the tensile force in the membrane.

The analytical solution to the square drumhead problem is expressed by

$w=A_{p q} \operatorname{sine}(p \pi x / H) \operatorname{sine}(q \pi y / H) \operatorname{sine}\left(f_{p q}^{t}\right)$

where $A_{p q}$ are arbitrary nonzero constants, $p$ and $q$ are positive, nonzero integers, $H$ is the length of a side of the square membrane, and, $f_{p q}$ are the resonant frequencies.

Thus, the modes are products of half sine waves over the planform of the structure. For a given $q$, mode displacement is an even function of $x$ when $p$ is odd and an odd function when $p$ is even.

Figure 2 shows the location of nodal vibration lines for the first 16 modes of the membrane. The nodal curves are straight lines that are orthogonal to each other.

Substitution of Eq. (2) in Eq. (1) prescribes that,

$$
f_{p q}^{2}=N \pi^{2}\left(p^{2}+q^{2}\right) / M
$$

where $M$ is the total mass of the surface.

Rayleigh's quotient can be evaluated by, 


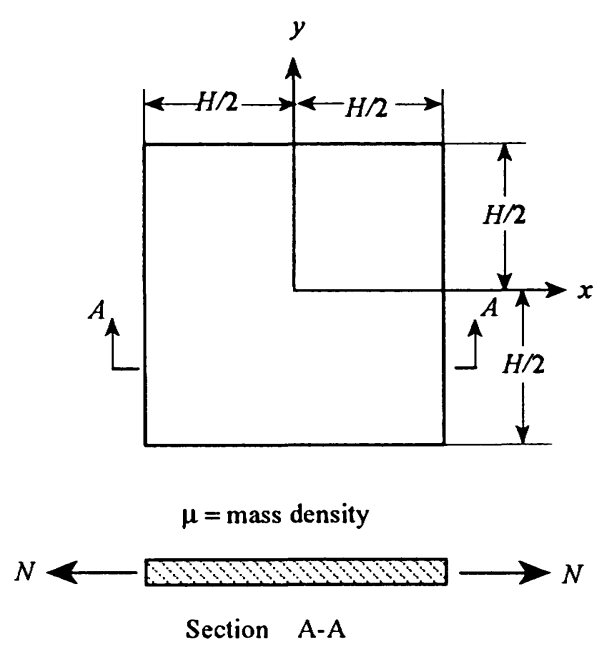

FIGURE 1 Drumhead geometry.

$$
\mathrm{RQ}_{p q}=1 / f_{p q}^{2}=1 / f_{q p}^{2}
$$

that is, Rayleigh's quotient is symmetric in $p$ and $q$.

The approximations made in arriving at the governing field equations are approximations of mathematical idealization. Because the mathematical model is a differential equation, it implies no FEA modeling approximations.

Converting the mathematical model to a finite number of equations of motion does incur FEA

THE PROBLEM: MODELING IN FEA OF A VIBRATING DRUMHEAD

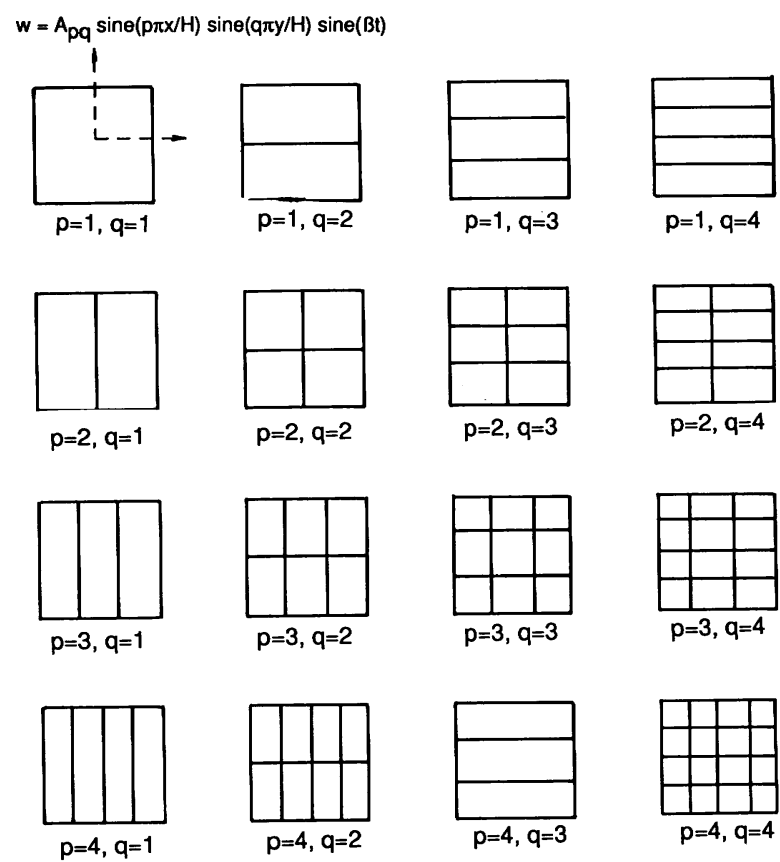

FIGURE 2 Vibration nodal lines for the square drumhead (first 16 modes). modeling approximations. The characteristics of the approximations are determined by the finite element model, the shapes of the element, allocation of the grid lines and nodes over the geometry of the structure and refinements of the finite element grid.

In the next section of this paper, we define the FEA model used in the experiments in more detail. Then the results of conventional FEA of the drumhead are displayed. The basis and results of adding curve fitting analysis to conventional analysis are presented. The sensitivity of analysis results as a function of the required accuracy and the number of vibration modes are estimated.

\section{FINITE ELEMENT MODELING APPROXIMATIONS}

Converting the mathematical model to a finite number of equations of motion incurs FEA modeling approximations. The characteristics of the approximations are determined by the finite element model, the shapes of the element, allocation of the grid lines and nodes over the geometry of the structure and refinements of the finite element grid.

We choose the following finite element modeling components:

1. The element model is based on hyperbolic variation of lateral displacement with the $x$ and $y$ coordinates. Thus, displacements have $C^{0}$ continuity across element boundaries.

2. The energy of displacements is calculated by integrating the energy density sampled at nodes of a $3 \times 3$ network of Gauss integration points. Then, because the displacement function is a polynomial, the quadrature yields the values of the strain and kinetic energies without additional modeling approximations.

Rayleigh's quotients for the structure are defined by,

$$
\mathrm{RQ}_{p q}=\mathrm{KE} / \mathrm{SE}=0.5 \sum \mathbf{w}^{\mathbf{T}} \mathbf{M w} /\left(0.5 \sum \mathbf{w}^{\mathbf{T}} \mathbf{M w}\right)
$$

where $\mathrm{KE}$ is the kinetic energy, $\mathrm{SE}$ is the strain energy, $\mathbf{w}$ is a normalized column eigenvector of lateral displacements, $\mathbf{K}$ is the symmetric fourth- 
order rank two element positive semidefinite stiffness matrix, $\mathbf{M}$ is the symmetric fourth-order rank four element positive definite mass matrix, and the summation, $\Sigma$, extends over all elements of the finite element model.

Each gridwork of elements constitutes a regular isogeometric subdivision of the original one by one grid of the drumhead. The sequence of finite element grids involves relative grid intervals, $h / H$, of $1 / 1,1 / 2,1 / 3,1 / 4,1 / 5 \ldots 1 / 19$.

The objective of the analysis is to evaluate Rayleigh's quotient to 1.3 digits of accuracy using a finite element model. This accuracy corresponds to tolerance of about 5\% error in analysis results: the accuracy aspired to in engineering practice.

For research purposes all calculations are performed using IEEE double precision arithmetic (about 15.6 digits of precision). The double-precision results of the conventional finite element analysis are truncated to single precision (6.92 digits of precision) for curve fitting analysis. The maximum loss of precision measured for all fittings was two digits. Therefore, the maximum number of digits of accuracy in the computer results is 4.9 digits. by,

The number of digits of accuracy is measured

$$
\begin{array}{r}
\mathrm{DA}=\operatorname{sygnum}\left(x_{1}-x_{2}\right) \log _{10}\left[\operatorname{abs}\left(x_{1}-x_{2}\right) /\right. \\
\left(0.5\left(x_{1}+x_{2}\right)\right]
\end{array}
$$

where DA is the number of decimal digits of accuracy, sygnum(. . .) takes the sign of a number, and, $x_{1}$ and $x_{2}$ are the values of the two numbers being compared. This definition implements the comparison of two independent estimates of a variable, $x$, without bias in the selection of the more meaningful estimate. In this paper, the $x_{1}$ are values of Rayleigh's quotient given by Eq. (3) and $x_{2}$ are the values of Rayleigh's quotient evaluated by FEA.

We measure the data processing resources needed for analysis by,

$$
\begin{aligned}
\mathrm{CI}=\Sigma_{i}\left(H / h_{i}\right)^{2}, & \text { with } i=1,2, \ldots \mathrm{NM}, \\
\text { and } \mathrm{NM} & =q *(q+1) / 2
\end{aligned}
$$

where $\mathrm{CI}$ is the calculations index, NM is the number of modes required by the analyst, and, $q \geq p$, or the roles of $p$ and $q$ are reversed. This definition implies that the resources associated with curve fitting analysis are negligible compared with those required in generating element stiffness and mass matrices and evaluating energies. Furthermore, we assume that the number of modes required by the analyst starts with mode $(1,1)$ and proceeds to mode $(q, q)$ by ordering by increasing $\left(p^{2}+q^{2}\right)$.

\section{A SURVEY OF THE FEA SOLUTION SPACE}

Table 1 lists the accuracy of 190 finite element solutions for 19 grids and 10 vibration modes. Because Rayleigh's quotient is symmetric in $p$ and $q$, the data of Table 1 are pertinent to analysis of the lowest 16 vibration modes and 19 grids and hence, 304 solutions.

Table 1 cells with more than a decimal number indicate spurious FEA estimates of quotient values. Cells containing $0 / 0$ are cases for which both the strain energy and the kinetic energy are zero because each FEA node is located on a vibration nodal line. Cells that discriminate more modes than the number of active degrees of freedom have linearly dependent modes that violate the orthogonality conditions.

Defining the number of active degrees of freedom as the number of generalized nodal displacements that take on nonzero values of displacements, we observe that linearly dependent modes are excluded when the FEA gridwork satisfies the criteria,

$$
H / h_{c}>\max (q, p)
$$

where $H / h_{c}$ is the coarseness limit on acceptable grid intervals, and $\max (p, q)$ indicates the maximum of $p$ or $q$.

The sign of the decimal number in each acceptable cell is positive when the FEA produces quotient estimates that are greater than the value given by Eq. (3). For $q \geq p$, these involve values of $H / h$ such that,

$$
H / h=1 * q, 2 * q, 3 * q . .
$$

Figure 3 displays the relation between accuracy and grid interval for modes $(1,1),(1,2)$, and $(4,4)$ developed by plotting results of the analyses of Table 1. These graphs portray the Sshaped convergence curves that are common in FEA applications (Melosh, 1990).

Figure 3 shows two curves for mode $(1,2)$ : one for the cells of Table 1 that involve positive DAs and one for cells with negative digits. Separating the data in this way results in two mono- 
Table 1. Digits of Accuracy of Rayleigh's Quotient

\begin{tabular}{rlrrrrrrrrr}
\hline \multicolumn{7}{c}{ H } & \multicolumn{1}{c}{ Number of $x$ and $y$ Half Sine Waves } \\
\hline 2.0 & $(1,1)$ & $(1,2)$ & $(2,2)$ & $(1,3)$ & $(2,3)$ & $(3,3)$ & $(1,4)$ & $(2,4)$ & $(3,4)$ & $(4,4)$ \\
3.0 & 1.0 & $-1,0$ & $0 / 0$ & $0 / 0$ & $0 / 0$ & $-0.2 \mathrm{a}$ & $0 / 0$ & $0 / 0$ & $0 / 0$ & $0 / 0$ \\
4.0 & 1.3 & $0.8 \mathrm{a}$ & $0.7 \mathrm{~b}$ & $-0.3 \mathrm{a}$ & $-0.5 \mathrm{~b}$ & -0.1 & $0 / 0$ & $0 / 0$ & $0 / 0$ & $0 / 0$ \\
5.0 & 1.5 & -0.9 & -0.8 & $-0.8 \mathrm{a}$ & -0.5 & -0.4 & $-0.2 \mathrm{a}$ & -0.2 & -0.1 & -0.1 \\
6.0 & 1.7 & 1.1 & 1.0 & $0.7 \mathrm{a}$ & $0.8 \mathrm{~b}$ & $0.7 \mathrm{c}$ & $-0.5 \mathrm{a}$ & $-0.6 \mathrm{~b}$ & $-0.9 \mathrm{c}$ & -0.3 \\
7.0 & 1.8 & -1.1 & -1.0 & -0.9 & -0.7 & -0.7 & -0.5 & -0.5 & -0.4 & -0.3 \\
8.0 & 1.9 & 1.4 & 1.3 & -1.0 & -1.0 & -0.8 & 0.7 & 0.8 & 1.1 & 0.7 \\
9.0 & 2.0 & -1.2 & -1.1 & 1.1 & 1.4 & 1.0 & -0.6 & 0.6 & -0.9 & -0.5 \\
10.0 & 2.1 & 1.6 & 1.5 & -1.1 & -1.1 & -0.9 & -0.8 & -0.9 & -0.8 & -0.8 \\
11.0 & 2.2 & -1.2 & -1.1 & -1.1 & -0.9 & -0.9 & -0.7 & -0.8 & -0.7 & -0.7 \\
12.0 & 2.2 & 1.7 & 1.6 & 1.3 & 1.4 & 1.3 & 1.1 & 1.1 & 1.1 & 1.0 \\
13.0 & 2.3 & -1.3 & -1.2 & -1.2 & -1.0 & -1.0 & -0.8 & -0.8 & -0.8 & -0.8 \\
14.0 & 2.4 & 1.8 & 1.8 & -1.2 & -1.2 & -1.0 & -1.0 & -1.0 & -1.0 & -1.0 \\
15.0 & 2.4 & -1.4 & -1.2 & 1.5 & 2.2 & 1.5 & -0.9 & -1.1 & -1.1 & -0.8 \\
16.0 & 2.5 & 2.0 & 1.9 & -1.3 & -1.2 & -1.1 & 1.3 & 1.4 & 1.3 & 1.3 \\
17.0 & 2.5 & -1.4 & -1.3 & -1.3 & -1.1 & -1.1 & -0.9 & -0.9 & -0.9 & -0.9 \\
18.0 & 2.6 & 2.1 & 2.0 & 1.7 & 1.7 & 1.6 & -1.1 & -1.3 & -1.3 & -1.1 \\
19.0 & 2.6 & -1.4 & -1.3 & -1.3 & -1.2 & -1.1 & -1.0 & -1.0 & -1.0 & -0.9 \\
\hline
\end{tabular}

Interpretation of cell values: $\mathrm{x} . \mathrm{x}=$ decimal digits of accuracy of Rayleigh's quotient; $0 / 0=$ both kinetic and strain energy are zero; $a, b$, and $c=$ repeated value for quotient: $-=$ estimated value of quotient is $>$ exact value.

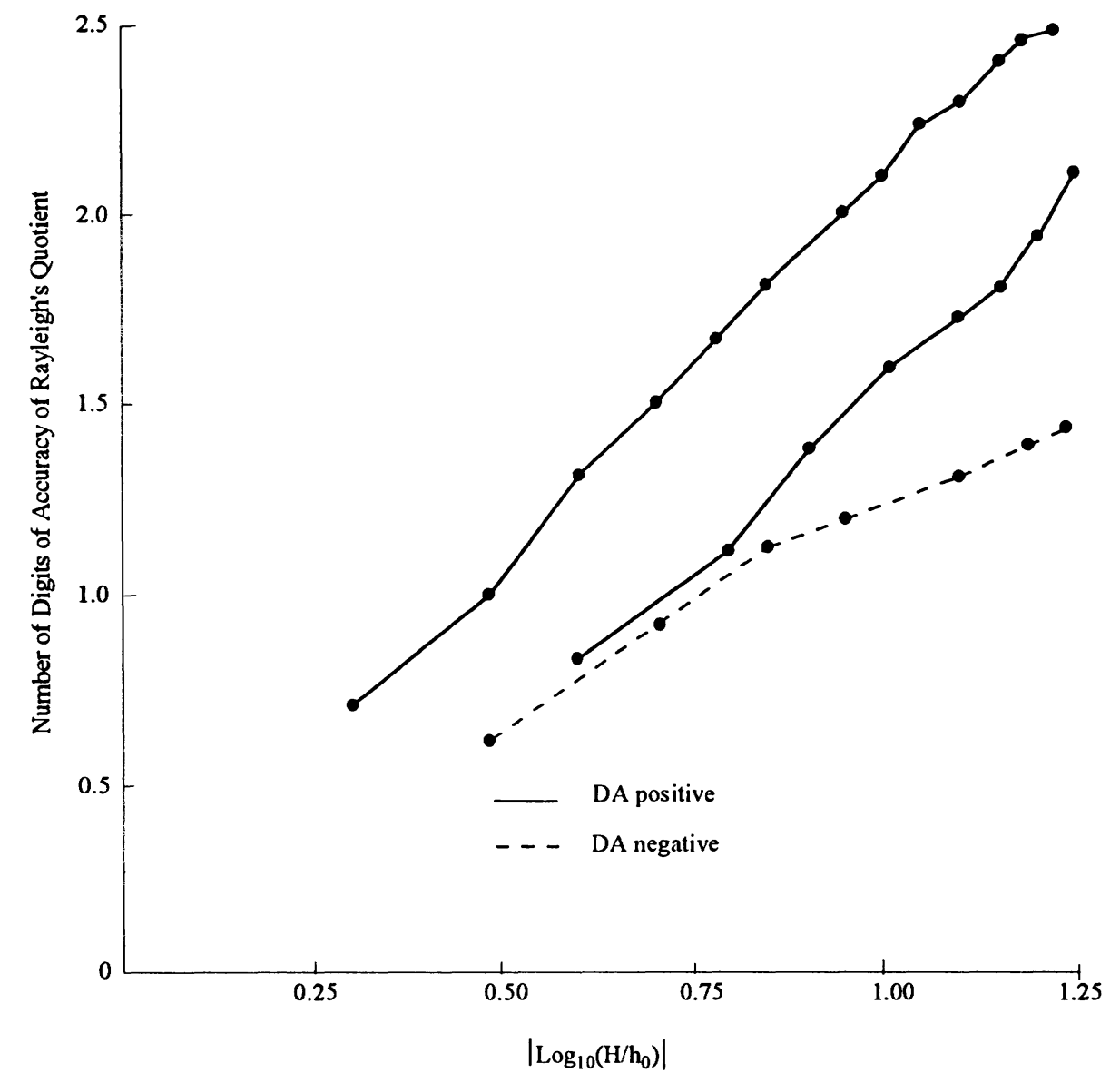

FIGURE 3 Convergence curves for vibrating drumhead. 
tonically converging sequences instead of one curve with an oscillating sequence of quotient accuracy values.

The convergence curve for mode $(1,1)$ also appears in Figure 3. This curve illustrates strictly monotonic convergence associates with mode (1, 1 ), that is, successively lower relative grid intervals result in successively higher values of DA and the slope of the curve (convergence rate) can only be zero when $h / H$ is 1 or 0 . Comparing the convergence rates for positive DA for mode (1, 2 ) with those for negative DA of mode $(1,2)$ suggests only a small change in the convergence rate.

The criteria of engineering accuracy is met when the number of finite elements per side of the membrane is given by,

$$
H / h \geq 4 \max (p, q)
$$

where $H / h$ is the reciprocal of the number of elements along a side of the square.

\section{CURVE FITTING ANALYSES}

We seek to enhance the estimates of Rayleigh's quotient by curve fitting the trial function,

$$
\mathrm{RQ}(h / H)=\mathrm{RQ}(h / H=0)+a(h / H)=\Sigma b_{i}(h / H)^{i}
$$

where $a$ and $b_{i}$ are curve fitting constants, and, the summation is over $i=2,3,4 \ldots, n$ with $n$ selected adaptively.

When $h / H$ is small enough, Eq. (11) represents a truncated Taylor series expansion of Rayleigh's quotient about $h / H=0$. This interpretation implies that RQ is a continuous function of $h / H$. Under the additional assumption that convergence of a sequence of Rayleigh quotients is asymptotic, we can limit the trial functions to cases for which $a=0$, that is,

$$
\mathrm{RQ}(h / H)=\mathrm{RQ}(h / H=0)+\sum b_{i}(h / H)^{i} .
$$

In this section we will examine use of the trial functions defined by Eq. (12) in representing the relationship between accuracy of the estimates of the quotient and relative grid interval.

Table 2 lists sampling of FEA grids for three different sampling strategies. Some of the quotient estimates are higher than the Eq. (3) value and some less.
Table 2. Enhanced Estimates of Rayleigh's Quotient

\begin{tabular}{lccc}
\hline & \multicolumn{3}{c}{ Cell Contents, Digits, RQ; Grid $H / h$} \\
\cline { 2 - 4 } Mode & $\begin{array}{c}\text { Conventional } \\
\text { Strategy }\end{array}$ & $\begin{array}{c}\text { Smallest } H / h \\
\text { Strategy }\end{array}$ & $\begin{array}{c}\text { Strictly Mono. } \\
\text { Strategy }\end{array}$ \\
\hline$(1,1)$ & 1.3 & 1.88 & 1.88 \\
& 4 & 2,3 & 2,3 \\
$(1,2)$ & 1.4 & 1.32 & 2.02 \\
& 8 & 3,4 & 4,6 \\
$(2,2)$ & 1.3 & 1.78 & 1.88 \\
& 8 & $4,5,6$ & 4,6 \\
$(1,3)$ & 1.3 & 1.38 & 2.78 \\
& 12 & 4,5 & 6,9 \\
$(2,3)$ & 1.4 & 1.46 & 2.04 \\
& 12 & $4,5,6$ & 6,9 \\
$(3,3)$ & 1.3 & 1.39 & 1.88 \\
& 12 & $4,5,6$ & 6,9 \\
$(1,4)$ & 1.3 & 1.6 & 1.93 \\
& 16 & $5,6,7,8$ & 8,12 \\
$(2,4)$ & 1.3 & 1.7 & 2.02 \\
& 16 & $5,6,7,8$ & 8,12 \\
$(3,4)$ & 1.4 & 1.82 & 2.03 \\
& 16 & $5,6,7,8$ & 8,12 \\
$(4,4)$ & 1.3 & 1.70 & 1.88 \\
& 16 & $5,6,7,8$ & 8,12 \\
\hline
\end{tabular}

The three strategies are:

1. Conventional FEA strategy: in this case, the grid is refined from the first acceptable $h / H$ values until the number of DA is greater than 1.3. In the notation of Eq. (12), $h / H$ is decreased and the data is fit by only the first term of the series.

2. Smallest $H / h$ strategy: the starting grid is the first acceptable grid for the mode of interest. $i$ is then increased by ones and curve fitting pursued until the accuracy exceeds 1.3. Thus, the value of $i$ is increased with $n=2,3, \ldots$ until the number of DA exceeds 1.3 .

3. Strictly monotonic strategy: the starting grid is the first acceptable grid for each mode. $i$ is then increased to the next higher value of $H / h$ that will produce a strictly monotonic convergence curve with a plus error.

The data of Table 2 indicates that each of the three strategies leads to Rayleigh quotients of engineering accuracy for the analytical solution, Eq. (3).

The conventional strategy furnishes quotients with the smallest range in the number of DA, 1.3 
Table 3. Calculations Index for Rayleigh's Quotients

\begin{tabular}{cccc}
\hline $\begin{array}{l}\text { Vibration } \\
\text { Mode }(p, q)\end{array}$ & $\begin{array}{c}\text { Conventional } \\
\text { Strategy }^{\mathrm{a}}\end{array}$ & $\begin{array}{c}\text { Small } H / h \\
\text { Strategy* }\end{array}$ & $\begin{array}{c}\text { Super Mono. } \\
\text { Strategy }^{\mathrm{a}}\end{array}$ \\
\hline$(1,1)$ & 16 & 13 & 13 \\
$(1,2)$ & 80 & 38 & 65 \\
$(2,2)$ & 144 & 115 & 117 \\
$(1,3)$ & 288 & 156 & 234 \\
$(2,3)$ & 432 & 233 & 351 \\
$(3,3)$ & 576 & 310 & 468 \\
$(1,4)$ & 832 & 484 & 676 \\
$(2,4)$ & 1088 & 658 & 884 \\
$(3,4)$ & 1344 & 832 & 1092 \\
$(4,4)$ & 1600 & 1006 & 1300 \\
\hline
\end{tabular}

aValue is the total number of finite elements in the models of the vibrating square membrane, required to attain $\mathrm{DA} \geq$ 1.3 for each mode.

to 1.4 . The strictly monotonic strategy produces the highest range of $D$ while meeting the 1.3 digit requirement: 1.88 to 2.7 .

Table 3 defines the values of calculations index as a function of the vibration mode and the FEA sampling strategy. Table 3 lists calculation indicies for the three grid refinement strategies for $\mathrm{NM}=1$ to 10 . These data indicate that index reductions using curve fitting analysis are better characterized by percentage reductions than by powers of 10 , that the smallest $H / h$ strategy tends to minimize the index (up to $62 \%$ lower than that of the conventional FEA), and that use of the strictly monotonic strategy does not minimize the index.

\section{SENSITIVITY OF THE CALCULATIONS INDEX}

The data of Table 4 define values of the calculations index for various DA and NM. The data suggest that the index is given by,

$$
\mathrm{CI}=12 * D A^{*} N M^{2} .
$$

We conclude that the calculations index increases linearly with the DA and quadratically with the NM required.
Table 4. Sensitivity of the Calculations Index

\begin{tabular}{llll}
\hline & \multicolumn{3}{c}{ Number of Digits of Accuracy } \\
Mode & \multicolumn{1}{c}{1.3} & 2.0 & \multicolumn{1}{c}{2.7} \\
\hline $\begin{array}{l}(1,1), 1 \text { mode } \\
(1,1)-(4,4),\end{array}$ & 16 & 81 & 400 \\
$\quad 10$ modes & & $89 * 10^{2}$ & $400 * 10^{2}$ \\
$(1,1)-(14,14)$, & $18 * 10^{4}$ & $89 * 10^{4}$ & $440 * 10^{4}$ \\
105 modes & & & \\
\hline
\end{tabular}

aValue is the total number of finite elements in the models of the vibrating square membrane, required to attain DA $\geq$ 1.3 for each mode.

\section{CONCLUSIONS}

The analyses of the vibrating square drumhead lead to the following conclusions:

1. Finite element analyses can produce unacceptable results when the grid is too coarse to represent the structural system. (Selective sampling of FEA results is a means for rendering oscillating convergence curves by curves that reflect strictly monotonic convergence.)

2. Curve fitting analysis reduces the calculations index for all cases of Table 3. Reductions were up to $52 \%$ of the index associated with conventional strategy analyses.

3 . The calculations index for drumhead analysis increases monotonically with increases in the NM and DA required. (The calculations index indicates that the data processing resources increase linearly with the DA and NM required by the analyst.)

It remains to verify which of the conclusions are valid for more general geometries than square elements and drumheads.

\section{REFERENCES}

Collatz, L., "Eigenvalue Problems," Handbook of Engineering Mechanics, Chap. 18, in W. Flugge, 1962, McGraw-Hill Book Company, New York.

Melosh, R. J., 1990, "Finite element analysis of convergence curves," Finite Elements in Analysis and Design, Vol. 7, pp. 115-121.

Young, D., "Continuous Systems," in W. Flugge, Handbook of Engineering Mechanics, Chap. 61, McGraw-Hill Book Company, New York. 

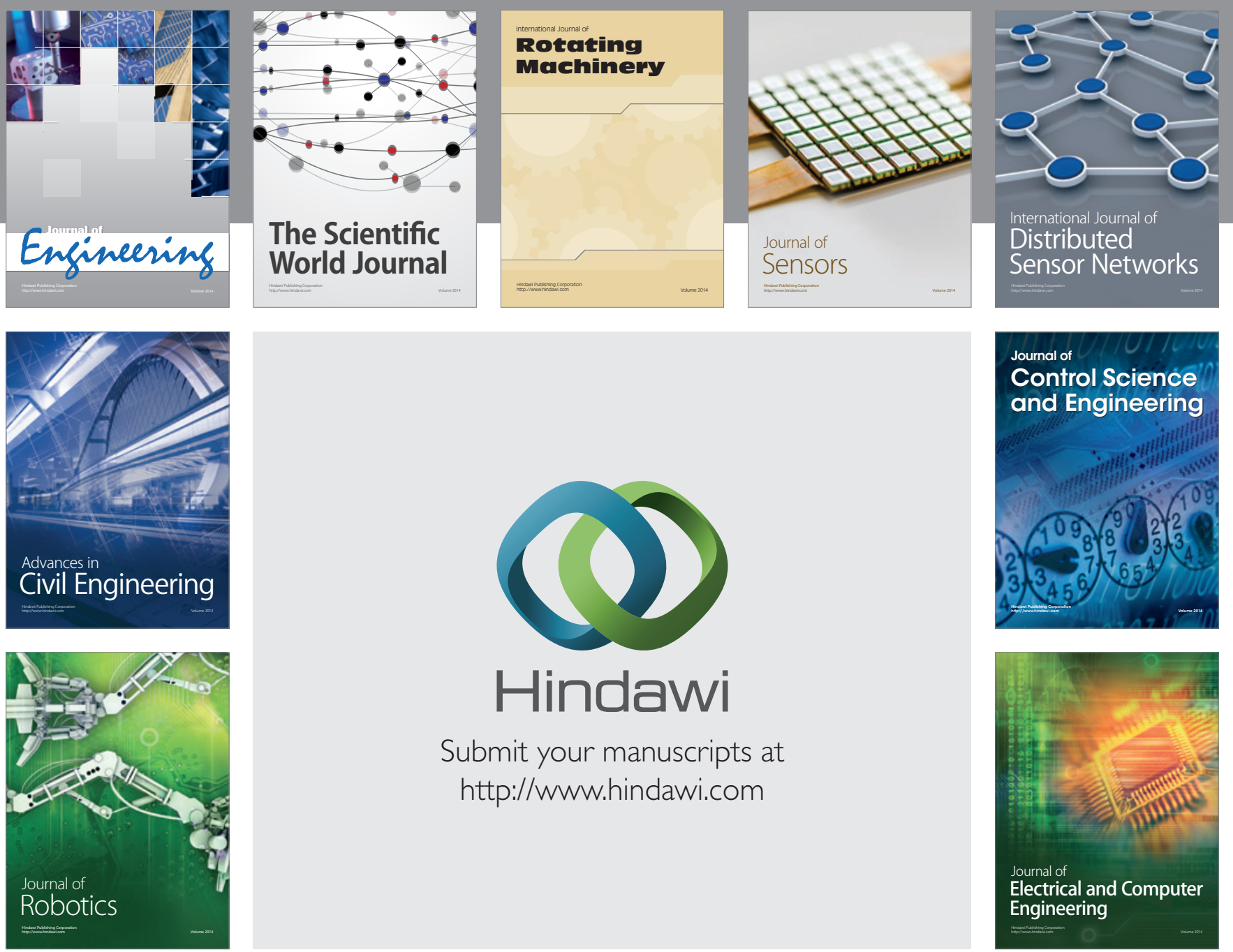

Submit your manuscripts at

http://www.hindawi.com
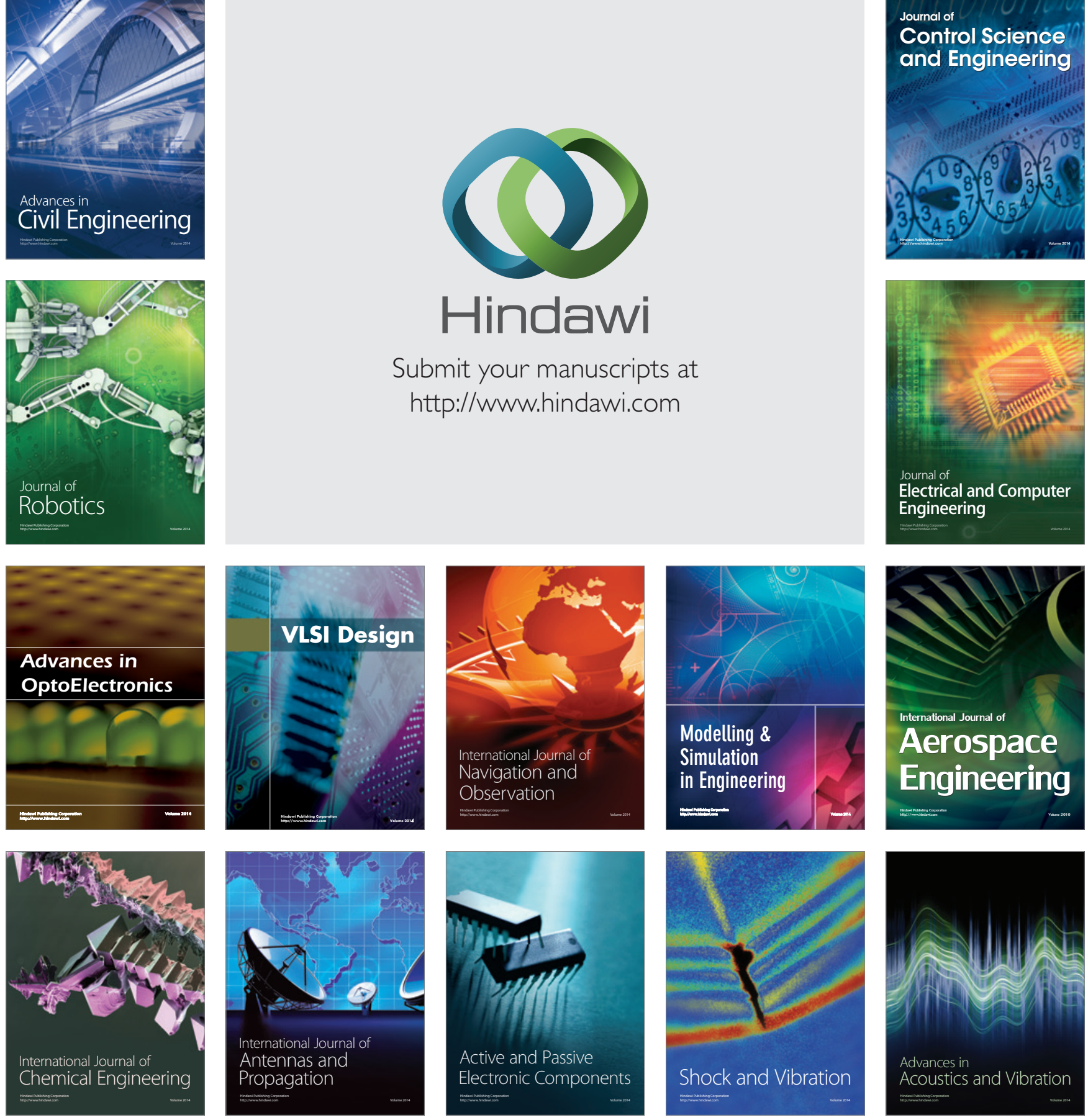